\title{
An Improved Method of Vehicle Driving Cycle Construction: A Case Study of Beijing
}

\author{
Zhenpo Wang ${ }^{1, \mathrm{a}}$, Yang $\mathrm{Li}^{1, \mathrm{~b}}$, Hao Luo ${ }^{1}$, Xue Xue \\ ${ }^{1}$ National Engineering Laboratory for Electric Vehicles, Beijing Institute of Technology, Beijing, \\ 100081, China \\ awangzhenpo@bit.edu.cn, by736790136@163.com
}

Keywords: Driving Cycle Development; Micro-trips; Principal Component Analysis; K-means Clustering Analysis

Abstract. The traditional vehicle driving cycle construction method is to use a random method to choose the cycle segments, which is lack of reliability and accuracy. In order to solve the problems of the traditional construction method, an improved methodology for developing a representative driving cycle for Beijing city was proposed in this paper. The methodology included four parts: data acquisition, kinematic sequences division, candidate segments classification and final segments selection. In the process of candidate cycle selection, we adopted a method based on the minimum characteristic parameters deviation to choose the final segments to construct driving cycle. The results indicate that this method can further improves the representativeness of the resultant driving cycle.

\section{Introduction}

In recent years, vehicle has become more and more popular in China. To quantify the energy consumption and pollutant emission of the vehicles, researchers developed standard driving cycles for relevant tests. A driving cycle is expressed as the speed-time curve, which is applied to show vehicle driving characters with certain limited conditions[1]. Both fuel economy and emission performance tests are relied on it, as well as the new car design[2]. Nevertheless, due to the late start of China's automobile industry, there is no official driving cycle to represent the real situation in China, and then the European test cycle (NEDC) is widely used. However, there are so many differences between the two country, such as road condition, geographical situation and vehicle performance[3]. Therefore, it's necessary and urgent to develop a practical driving cycle that represent China's realistic situation. At the meantime, the methodology of cycle construction also becomes a popular research areas.

A large amount of methods were employed on cycle construction, among which, the micro-trips is most widely used both domestic and overseas[4]. In the traditional method, Principal Component Analysis (PCA)[5] and K-means Cluster Analysis[6] are usually applied for characteristic parameters analysis of micro-trips. After that, a random selection process is adopted for synthesizing the candidate cycle trips, which will affect the accuracy and precision of the final cycle curve. This paper aims to address this issue by providing a feasible method to assure that the selected trips can furthest represent the real driving features. The final result shows the improved method produces a much more rational driving cycle.

\section{The Traditional Method for Cycle Construction}

The traditional method is based on a large amount of on-board data. After data processing, the speed-time curve is divided into lots of micro-trips. Principal Component Analysis is applied for mapping multidimensional characteristic parameters of all the micro-trips into lower dimensions with minimal loss of information[7], then K-means Cluster Analysis is employed to classify all the micro-trips into different groups according to their own features. At last, the final cycle are obtained by random selection, and the process flow diagram is shown in Fig. 1. Many researchers tend to use this approach because it decomposes the data into fragments so that the process of calculation and analysis become not that much complicated[8]. 

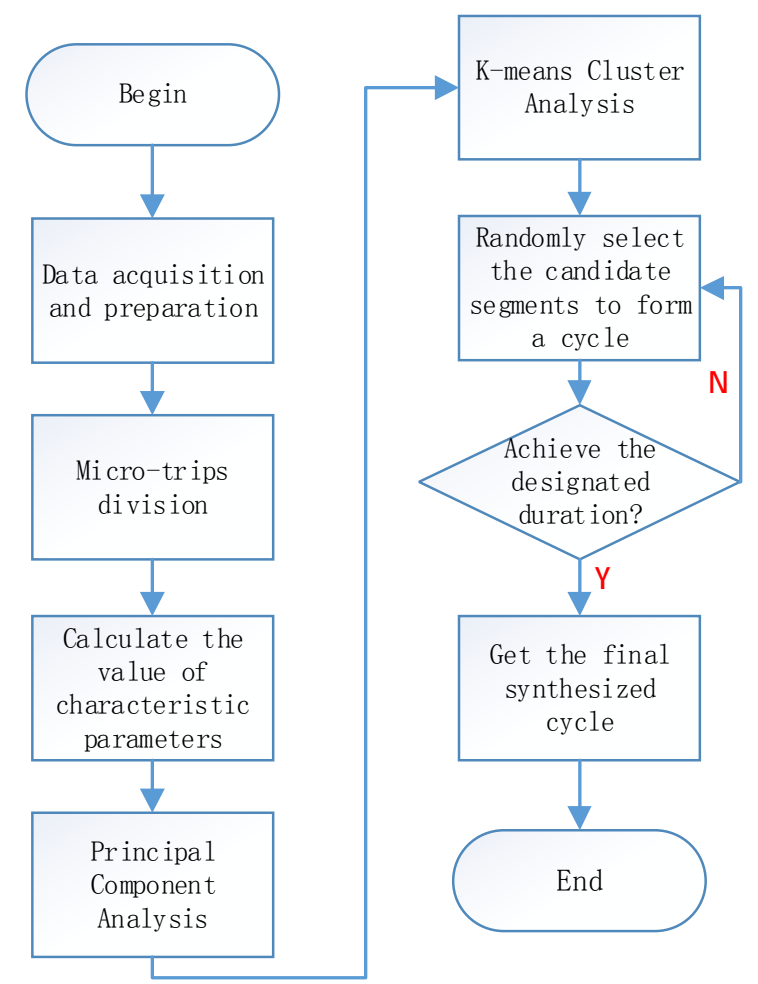

Fig. 1 Process of the Traditional Method for Cycle Construction

The micro-trips is based on the theory of kinematic sequences, which is performed by dividing trips into sequences between idle points[9]. A standard kinematic sequence usually lasts for 20 seconds at least and comprises four parts as follows:

Idling mode: The speed is zero, while the car is not shutoff, waiting for starting or recover running;

Accelerating mode: Continuous process whose acceleration is larger than $0.15 \mathrm{~m} / \mathrm{s}^{2}$;

Decelerating mode: Continuous process whose deceleration is smaller than $-0.15 \mathrm{~m} / \mathrm{s}^{2}$;

Cruising mode: continuous process whose absolute value of acceleration or deceleration is less than $0.15 \mathrm{~m} / \mathrm{s}^{2}$ while the speed is not zero.

For the purpose of analyzing the vehicle driving situation, several characteristic parameters such as duration, distance traveled and average speed which is shown in Table 1 and Table 2, are adopted for the succeeding procedures.

Table 1 Driving Characteristic Parameters

\begin{tabular}{c|lll}
\hline $\begin{array}{c}\text { Sequence } \\
\text { No. }\end{array}$ & Sign & Description & Unit \\
\hline 1 & $\mathrm{~T}$ & Duration & $\mathrm{s}$ \\
2 & $\mathrm{~S}$ & Distance & $\mathrm{m}$ \\
3 & $\mathrm{~T}_{\mathrm{i}}$ & Idle time & $\mathrm{s}$ \\
4 & $\mathrm{~T}_{\mathrm{a}}$ & Acceleration time & $\mathrm{s}$ \\
5 & $\mathrm{~T}_{\mathrm{d}}$ & Deceleration time & $\mathrm{s}$ \\
6 & $\mathrm{~T}_{\mathrm{e}}$ & Cruise time & $\mathrm{s}$ \\
7 & $\mathrm{~V}_{\max }$ & Maximum speed & $\mathrm{km} / \mathrm{h}$ \\
8 & $\mathrm{~V}_{\mathrm{m}}$ & Average speed & $\mathrm{km} / \mathrm{h}$ \\
9 & $\mathrm{~V}_{\mathrm{mr}}$ & Average running speed (excluding the idle periods) & $\mathrm{km} / \mathrm{h}$ \\
10 & $\mathrm{~V}_{\mathrm{s}}$ & Standard speed deviation & $\mathrm{km} / \mathrm{h}$ \\
11 & $\mathrm{~A}_{\max }$ & Maximum acceleration & $\mathrm{m} / \mathrm{s} 2$ \\
12 & $\mathrm{~A}_{\mathrm{m}}$ & Average acceleration & $\mathrm{m} / \mathrm{s} 2$ \\
13 & $\mathrm{D}_{\max }$ & Maximum deceleration (absolute value) & $\mathrm{m} / \mathrm{s} 2$ \\
14 & $\mathrm{D}_{\mathrm{m}}$ & Average deceleration (absolute value) & $\mathrm{m} / \mathrm{s} 2$ \\
15 & $\mathrm{~A}_{\mathrm{s}}$ & Standard acceleration deviation & $\mathrm{m} / \mathrm{s} 2$ \\
\hline
\end{tabular}


Table 2 Distribution Characteristic Parameters

\begin{tabular}{c|llr}
\hline Sequence No. & Sign & Description & Unit \\
\hline 1 & $\mathrm{P}_{\mathrm{i}}$ & Idle proportion & $\%$ \\
2 & $\mathrm{P}_{\mathrm{a}}$ & Acceleration proportion & $\%$ \\
3 & $\mathrm{P}_{\mathrm{d}}$ & Deceleration proportion & $\%$ \\
4 & $\mathrm{P}_{\mathrm{e}}$ & Cruise proportion & $\%$ \\
5 & $\mathrm{P}_{0-10}$ & Distribution proportion of speed within $0-10 \mathrm{~km} / \mathrm{h}$ & $\%$ \\
6 & $\mathrm{P}_{10-20}$ & Distribution proportion of speed within 10-20km $/ \mathrm{h}$ & $\%$ \\
7 & $\mathrm{P}_{20-30}$ & Distribution proportion of speed within 20-30km/h & $\%$ \\
8 & $\mathrm{P}_{30-40}$ & Distribution proportion of speed within 30-40km/h & $\%$ \\
9 & $\mathrm{P}_{40-50}$ & Distribution proportion of speed within 40-50km/h & $\%$ \\
10 & $\mathrm{P}_{50-60}$ & Distribution proportion of speed within 50-60km/h & $\%$ \\
11 & $\mathrm{P}_{60-70}$ & Distribution proportion of speed within $60-70 \mathrm{~km} / \mathrm{h}$ & $\%$ \\
12 & $\mathrm{P}_{70-80}$ & Distribution proportion of speed within 70-80km/h & $\%$ \\
13 & $\mathrm{P}_{80}$ & Distribution proportion of speed over 80km/h & $\%$ \\
14 & $\mathrm{~A}_{-2}$ & Distribution proportion of acceleration below $-2 \mathrm{~m} / \mathrm{s}^{2}$ & $\%$ \\
15 & $\mathrm{~A}_{-2 \sim-1.5}$ & Distribution proportion of acceleration within $-2 \sim-1.5 \mathrm{~m} / \mathrm{s}^{2}$ & $\%$ \\
16 & $\mathrm{~A}_{-1.5 \sim-1}$ & Distribution proportion of acceleration within $-1.5 \sim-1 \mathrm{~m} / \mathrm{s}^{2}$ & $\%$ \\
17 & $\mathrm{~A}_{-1 \sim 0.5}$ & Distribution proportion of acceleration within $-1 \sim-0.5 \mathrm{~m} / \mathrm{s}^{2}$ & $\%$ \\
18 & $\mathrm{~A}_{-0.5-0.5}$ & Distribution proportion of acceleration within $-0.5 \sim 0.5 \mathrm{~m} / \mathrm{s}^{2}$ & $\%$ \\
19 & $\mathrm{~A}_{0.5 \sim 1}$ & Distribution proportion of acceleration within $0.5 \sim 1 \mathrm{~m} / \mathrm{s}^{2}$ & $\%$ \\
20 & $\mathrm{~A}_{1 \sim 1.5}$ & Distribution proportion of acceleration within $1 \sim 1.5 \mathrm{~m} / \mathrm{s}^{2}$ & $\%$ \\
21 & $\mathrm{~A}_{1.5 \sim 2}$ & Distribution proportion of acceleration within $1.5 \sim 2 \mathrm{~m} / \mathrm{s}^{2}$ & $\%$ \\
22 & $\mathrm{~A}_{2}$ & Distribution proportion of acceleration over $2 \mathrm{~m} / \mathrm{s}$ & \\
\hline
\end{tabular}

\section{The Traditional Method Improvements}

As what have been mentioned above, the traditional driving cycle construction method is to use a random method to select the final segments, which is lack of reliability so it can not reflect the realistic situation. In view of this, a more scientific and rational approach is put forward as follows:

(1) Assume that all micro-trips have been divided into $n$ categories, the number of characteristic parameters is $p$ and $m_{k}$ is the number of micro-trips segments for the $k$-th category $(k=1,2,3 \ldots, n)$. Firstly, we need to normalize all the parameters before we use them in the succeeding calculation because they have different dimensions. The normalizing formulas are shown in Eq. 1. :

$$
z_{i j, k}=\left(x_{i j, k}-\min x_{j, k}\right) /\left(\max x_{j, k}-\min x_{j, k}\right) \quad\left(i=1,2,3, \mathrm{~K}, m_{k}\right)
$$

where $x_{i j, k}$ is the value of $j$-th parameter of the $i$-th segments in the $k$-th category, max $x_{j, k}$ and $\min x_{j, k}$ are the maximum and minimum value of the $j$-th parameter among all segments in the $k$-th category, Separately. $z_{i j, k}$ is the characteristic parameter values after normalization.

(2) Calculate the sum of parameters of each segments of $k$-th category by using Eq. 2 :

$$
z_{i, k}=\sum_{j=1}^{p} z_{i j, k} \quad\left(i=1,2,3, \mathbf{K}, m_{k}\right)
$$

(3)Normalize the average value of the $j$-th parameter among all segments in the $k$-th category and calculate the sum of the normalized parameters by using Eq. 3: 


$$
Y_{k}=\sum_{j=1}^{p}\left(\bar{x}_{j, k}-\min x_{j, k}\right) /\left(\max x_{j, k}-\min x_{j, k}\right)
$$

where $\bar{x}_{j, k}$ is the average value of the $j$-th parameter among all segments in the $k$-th category. $Y_{k}$ is the sum of the normalized parameters of the $k$-th category.

(4) Calculate the duration time of each category by using Eq. 4:

$$
T_{k}=\frac{\sum_{i=1}^{m_{k}} T_{i, k}}{\sum_{k=1}^{n} \sum_{i=1}^{m_{k}} T_{i, k}} \times \mathrm{T}_{f}
$$

where $T_{k}$ is the duration time of $k$-th category, $T_{i, k}$ is the duration time of $i$-th segments in the $k$-th category, $T_{f}$ is the duration time of final vehicle driving cycle.

(5) Calculate the absolute value of the deviation between $z_{i, k}$ and $Y_{k}$, select the segments of each category from the smallest value to the biggest till the duration time is greater than or equal to $T_{k}$.

(6) Combine the selected segments of each category to construct the final vehicle driving cycle.

\section{Empirical Analysis}

In order to verify the validity of improved approach, we use on-board measurement method to collect the data from 10 cars who run in Beijing typical road condition for 30 days. Through the PCA and K-means Cluster Analysis, the micro-trips are divided into 3 categories. Select the segments by using the proposed improved method, the final vehicle driving cycle curve is shown in Fig.2. The final cycle curve includes 11 micro-trip segments and lasts for 1237 seconds. Table 3 shows the deviation between the overall data and cycle data. It can be seen that the deviations are controlled within $10 \%$.

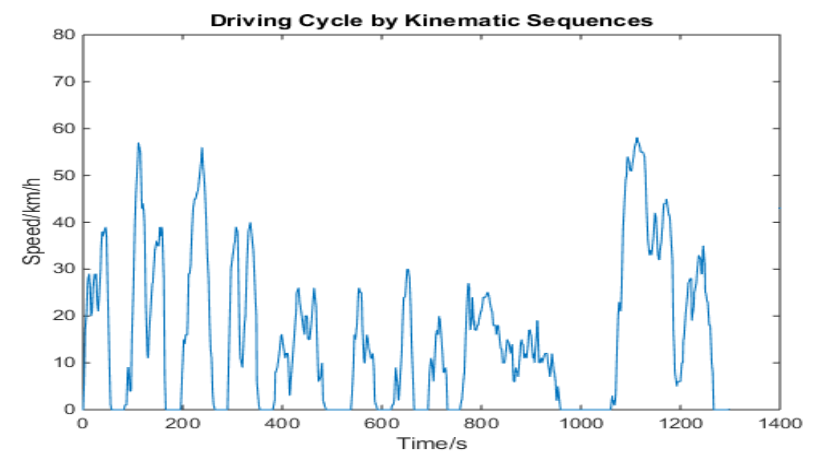

Fig. 2 Final Driving Cycle

Table 3 Distribution Characteristic Parameters

\begin{tabular}{c|ccc}
\hline Parameters & The Overall Data & Final Cycle & Error Rate \\
\hline $\mathrm{V}_{\mathrm{m}}$ & 17.353 & 16.560 & $4.56 \%$ \\
$\mathrm{~V}_{\mathrm{mr}}$ & 22.134 & 22.204 & $0.32 \%$ \\
$\mathrm{~V}_{\mathrm{s}}$ & 37.408 & 39.836 & $6.49 \%$ \\
$\mathrm{~A}_{\mathrm{m}}$ & 0.599 & 0.561 & $6.40 \%$ \\
$\mathrm{D}_{\max }$ & 0.599 & 0.633 & $5.57 \%$ \\
$\mathrm{D}_{\mathrm{m}}$ & 0.733 & 0.737 & $0.55 \%$ \\
$\mathrm{~A}_{\mathrm{s}}$ & 0.285 & 0.260 & $8.77 \%$ \\
$\mathrm{P}_{\mathrm{i}}$ & 0.252 & 0.260 & $3.21 \%$ \\
$\mathrm{P}_{\mathrm{a}}$ & 0.229 & 0.226 & $1.12 \%$ \\
$\mathrm{P}_{\mathrm{d}}$ & 0.234 & 0.253 & $8.33 \%$ \\
$\mathrm{P}_{\mathrm{e}}$ & 17.353 & 16.560 & $4.56 \%$ \\
\hline
\end{tabular}




\section{Results Analysis}

Comparison between Traditional Method and Improved Method. In order to compare the rationality and accuracy between traditional method and improved method, we construct the vehicle driving cycle by using traditional method, which is shown in Fig.3. Table 4 shows the driving characteristic parameters of overall data, improved method and traditional method. Fig. 4 shows the deviation analysis for the traditional method and improved method.

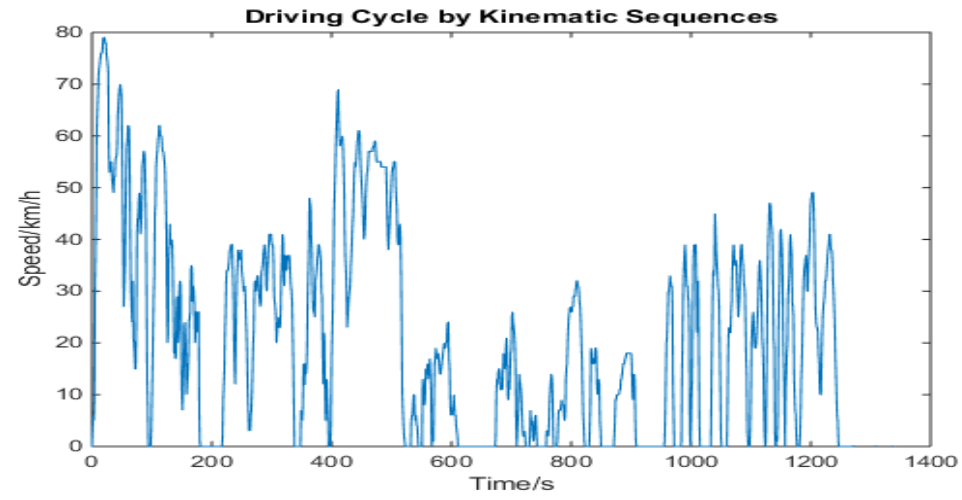

Fig. 3 Driving Cycle by Traditional Method

Table 4 Driving Characteristic Parameter Comparison

\begin{tabular}{c|ccc}
\hline Parameters & The Overall Data & Improved Method & Traditional Method \\
\hline $\mathrm{T}$ & 202655 & 1237 & 1234 \\
$\mathrm{~S}$ & 1086.155 & 6.656 & 6.697 \\
$\mathrm{~T}_{\mathrm{i}}$ & 57841 & 300 & 256 \\
$\mathrm{~T}_{\mathrm{a}}$ & 51126 & 345 & 359 \\
$\mathrm{~T}_{\mathrm{d}}$ & 46313 & 292 & 347 \\
$\mathrm{~T}_{\mathrm{e}}$ & 47375 & 300 & 272 \\
$\mathrm{~V}_{\max }$ & 86 & 58 & 62 \\
$\mathrm{~V}_{\mathrm{m}}$ & 17.353 & 16.560 & 16.770 \\
$\mathrm{~V}_{\mathrm{mr}}$ & 22.134 & 22.204 & 20.502 \\
$\mathrm{~V}_{\mathrm{s}}$ & 37.408 & 39.836 & 35.913 \\
$\mathrm{~A}_{\max }$ & 11.389 & 1.667 & 2.222 \\
$\mathrm{~A}_{\mathrm{m}}$ & 0.599 & 0.561 & 0.544 \\
$\mathrm{D}_{\max }$ & 8.611 & 3.889 & 2.778 \\
$\mathrm{D}_{\mathrm{m}}$ & 0.599 & 0.633 & 0.543 \\
$\mathrm{~A}_{\mathrm{s}}$ & 0.733 & 0.737 & 0.669 \\
\hline
\end{tabular}

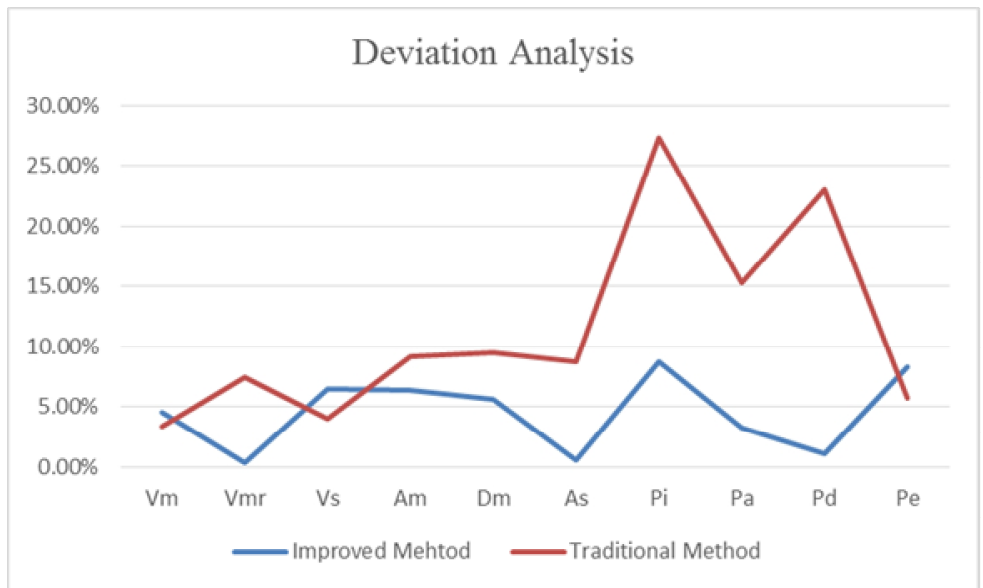

Fig. 4 Deviation Analysis for the two Methods 
As can be seen from Table 4 and Fig.4, the deviations of the improved method are smaller, especially for such parameters as $V_{m r}, A_{s}, P_{d}$. On the contrary, the deviations of the traditional method are much bigger, some parameter deviations are even more than $20 \%$. The comparison result shows that the improved method bring a far more accurate results than traditional method.

The distribution of the characteristic parameters and driving status can also reflect the similarity between the overall data and the final driving cycle. Therefore, we calculate the results of the distribution of the characteristic parameters and driving status of the overall data, improved method and traditional method, which are shown in Table 5 and Fig.5, separately.

Table 5 Distribution Characteristic Parameter Comparison

\begin{tabular}{c|ccc}
\hline Parameters & The Overall Data & Improved Cycle & Traditional Cycle \\
\hline $\mathrm{P}_{\mathrm{i}}$ & $28.54 \%$ & $26.04 \%$ & $20.75 \%$ \\
$\mathrm{P}_{\mathrm{a}}$ & $25.23 \%$ & $26.04 \%$ & $29.09 \%$ \\
$\mathrm{P}_{\mathrm{d}}$ & $22.85 \%$ & $22.60 \%$ & $28.12 \%$ \\
$\mathrm{P}_{\mathrm{e}}$ & $23.38 \%$ & $25.32 \%$ & $22.04 \%$ \\
$\mathrm{P}_{0-10}$ & $43.75 \%$ & $45.25 \%$ & $33.33 \%$ \\
$\mathrm{P}_{10-20}$ & $17.55 \%$ & $18.79 \%$ & $22.22 \%$ \\
$\mathrm{P}_{20-30}$ & $14.13 \%$ & $14.19 \%$ & $14.81 \%$ \\
$\mathrm{P}_{30-40}$ & $11.41 \%$ & $11.33 \%$ & $7.41 \%$ \\
$\mathrm{P}_{40-50}$ & $7.53 \%$ & $7.01 \%$ & $22.22 \%$ \\
$\mathrm{P}_{50-60}$ & $3.76 \%$ & $3.43 \%$ & $0.00 \%$ \\
$\mathrm{P}_{60-70}$ & $1.44 \%$ & $0.00 \%$ & $0.00 \%$ \\
$\mathrm{P}_{70-80}$ & $0.41 \%$ & $0.00 \%$ & $0.00 \%$ \\
$\mathrm{P}_{80}$ & $0.01 \%$ & $0.00 \%$ & $0.00 \%$ \\
$\mathrm{~A}_{-2}$ & $0.82 \%$ & $1.47 \%$ & $0.50 \%$ \\
$\mathrm{~A}_{-2 \sim 1.5}$ & $1.91 \%$ & $1.22 \%$ & $1.74 \%$ \\
$\mathrm{~A}_{-1.5 \sim-1}$ & $5.56 \%$ & $3.91 \%$ & $6.97 \%$ \\
$\mathrm{~A}_{-1 \sim 0.5}$ & $15.27 \%$ & $16.14 \%$ & $13.68 \%$ \\
$\mathrm{~A}_{-0.5-0.5}$ & $50.92 \%$ & $52.32 \%$ & $52.24 \%$ \\
$\mathrm{~A}_{0.5 \sim 1}$ & $18.70 \%$ & $19.80 \%$ & $17.91 \%$ \\
$\mathrm{~A}_{1 \sim 1.5}$ & $5.10 \%$ & $4.40 \%$ & $4.98 \%$ \\
$\mathrm{~A}_{1.5 \sim 2}$ & $1.31 \%$ & $0.73 \%$ & $1.74 \%$ \\
$\mathrm{~A}_{2}$ & $0.42 \%$ & $0.00 \%$ & $0.25 \%$ \\
\hline
\end{tabular}

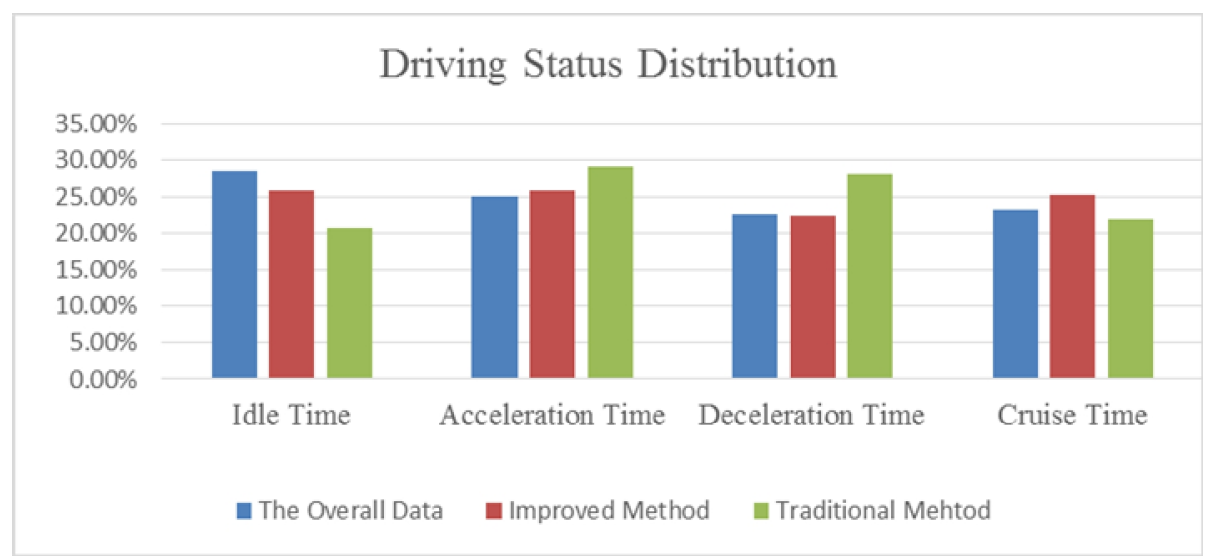

Fig. 5 Driving Status Distribution

As can be seen from Table 5 and Fig.5, the distribution of the characteristic parameters and driving status of the improved method are closer to the overall data, which means it has a higher similarities with the overall data. That is to say, the driving cycle constructed by the improved method is far more representative for the real driving situation.

Driving Characteristic Analysis. On the basis of the preceding chapters, a driving cycle for passenger vehicles in Beijing is developed. It can be realized from the statistical data that the driving 
situation in Beijing is terrible for the following points: (1) Idle time proportion is up to $26 \%$ and acceleration and deceleration occurs frequently; (2) Average speed is only $16.56[\mathrm{~km} / \mathrm{h}]$, and average running speed is $22.2[\mathrm{~km} / \mathrm{h}]$; (3) Speed is mainly distributed on the range of $0-30[\mathrm{~km} / \mathrm{h}]$ and its ratio is up to $78 \%$.

The data reveals that traffic jam is usually appears and lasts a long time in Beijing, the vehicles always start and stop sharply in the driving process, which is in accordance with the actual situation in Beijing.

\section{Conclusions}

(1) In this paper, an improved method based on the minimum characteristic parameters deviation was developed in the process of candidate cycle selection. This approach can solve the problem of inaccuracy and randomness caused by the traditional method effectively. From the statistical data obtained from the final vehicle driving cycle constructed by improved method, it can be concluded that the improved method is able to promote the precision and validity of the vehicle driving cycle.

(2) Judging by the figures and statistical data of the developed driving cycle for passenger vehicles in Beijing, it can be easily found that the traffic in Beijing has the features of heavy congestion, frequent acceleration and deceleration and lower average speed. The traffic condition and vehicle driving situation need to be improved.

\section{Acknowledgements}

This work was financially supported by the "New Energy Automobile Application Mechanism Research Based on the Integration of Beijing-Tianjin-Hebei Region” project (1700036111503).

\section{References}

[1] H.L. Ma, J.X. Ding, G.L. Wang, S. Guo, S.B. Cai: Chinese Journal Of Automotive Engineering (In Chinese), Vol. 4-1 (2014), p. 56-60.

[2] S. Ho, Y.D. Wong, V.W. Chang: Atmospheric Environment, Vol. 97 (2014), p. 353-362.

[3] Q.D. Wang, H. Huo, K.B. He, Z.L. Yao, Q. Zhang: Transportation Research Part D: Transport and Environment, Vol. 13-5 (2008), p. 289-297.

[4] Q. Shi, Y.B. Zheng: China Mechanical Engineering (In Chinese), Vol. 22-9 (2011), p.1123-1127.

[5] Y.J. Liu, J.Y. Li, B. Shen: International Journal of Control and Automation, Vol. 7-3 (2014), p. 125-136.

[6] A. Fotouhi, M. Montazeri-Gh: Scientia Iranica, Vol. 20-2 (2013), p. 286-293.

[7] M. Andrejic, N. Bojovic, M. Kilibarda: Expert Systems with Applications, Vol. 40-40 (2013), p. 3926-3933.

[8] J. Wang, Q.N. Wang, X.H. Zeng, P.Y. Wang, J.N. Wang: International Journal of Automotive Technology, Vol. 16-4 (2015), p. 685-695.

[9] J. Lina, D.A. Niemeier: Atmospheric Environment, Vol. 36-38 (2002), p. 5759-5770. 